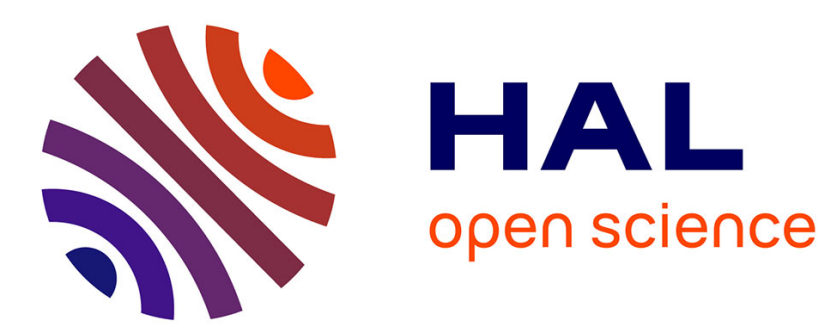

\title{
Automatic vehicle perpendicular parking design using saturated control
}

\author{
Plamen Petrov, Fawzi Nashashibi
}

\section{To cite this version:}

Plamen Petrov, Fawzi Nashashibi. Automatic vehicle perpendicular parking design using saturated control. 2015 IEEE Jordan Conference on Applied Electrical Engineering and Computing Technologies (AEECT), IEEE Jordan section, Nov 2015, Amman, Jordan. 10.1109/AEECT.2015.7360566 . hal01323011

\section{HAL Id: hal-01323011 \\ https://inria.hal.science/hal-01323011}

Submitted on 29 May 2016

HAL is a multi-disciplinary open access archive for the deposit and dissemination of scientific research documents, whether they are published or not. The documents may come from teaching and research institutions in France or abroad, or from public or private research centers.
L'archive ouverte pluridisciplinaire $\mathbf{H A L}$, est destinée au dépôt et à la diffusion de documents scientifiques de niveau recherche, publiés ou non, émanant des établissements d'enseignement et de recherche français ou étrangers, des laboratoires publics ou privés. 


\section{Automatic Vehicle Perpendicular Parking Design Using Saturated Control}

\author{
Plamen Petrov \\ Faculty of Mechanical Engineering \\ Technical University of Sofia \\ Sofia, Bulgaria \\ ppetrov@tu-sofia.bg
}

\author{
Fawzi Nashashibi \\ Robotics \& Intelligent Transportation Systems (RITS) \\ INRIA - Rocquencourt \\ Domaine de Voluceau, France \\ fawzi.nashashibi@inria.fr
}

\begin{abstract}
This paper considers the perpendicular reverse parking problem of front wheel steering vehicles. Relationships between the widths of the parking aisle and the parking place, as well as the parameters and the starting position of the vehicle for planning a collision-free reverse perpendicular parking in one maneuver are presented. A nonlinear saturated (tanh-type) feedback steering controller for straight-line tracking is proposed and evaluated. It is demonstrated that the saturated controller, which is continuous, achieves quick steering can be successfully used in solving parking problems. Simulation results and first experimental tests confirm the effectiveness of the proposed control scheme.
\end{abstract}

Keywords-automatic parking; perpendicular parking; path planning; saturated control

\section{INTRODUCTION}

The perpendicular parking is efficient and economical because it accommodates the most vehicles per linear meter [1]. However, due to the special constraint environments, much attention and driving experience is needed to control the vehicle, and this parking maneuver may be a difficult task. For this reason, automated operation attracts significant attention from research view point, as well, and from the automobile industry. One of the difficulties in achieving automatic parking is the narrow operating place for collision-free motion of the vehicle, and planning of optimal trajectories is often used in the applications. In [2], an optimal stopping algorithm was designed for parking using an approach combining an occupancy grid with planning optimal trajectories for collision avoidance. The geometry of the perfect parallel parking maneuver is presented in [3]. In [4], a practical reverse parking maneuver planner is given. A trajectory planning method based on forward path generation and backward tracking algorithm, especially suitable for backward parking situations is reported in [5]. A car parking control using trajectory tracking controller is presented in [6]. In [7], a saturated feedback control for an automated parallel parking assist system is reported. In recent years, automatic parking systems have been also developed by several automobile manufacturers $[9,10]$.

In this paper, we deal with the problems of geometric collision-free path planning and feedback steering control for automatic perpendicular reverse parking. An important condition to achieve effective collision-free parking maneuver is to determine a convenient start position of the vehicle depending on the widths of the parking aisle and the parking place, as well as the dimensions and the steering capabilities of the car. Geometric path planning based on admissible circular arcs within the available spot is presented in order to steer the vehicle in the direction of the parking place in one maneuver. A saturated (tanh-type) feedback control is proposed which achieves quick steering in tiny spots and stabilizes (practical stabilization) the vehicle in the parking place. The parking problem is seen as an extension of the tracking problem, since the vehicle has to track a straight line which passes through the goal position in the parking space and is parallel to the parking place with varying velocity depending of the position of the vehicle with respect to the goal position. The rest of the paper is organized as follows: In Section II, geometric considerations for planning perpendicular reverse parking in one maneuver are presented. In Section III, a saturated feedback steering controller is described. Simulation results and first experimental tests are reported in Section IV. Section V concludes the paper.

\section{Collision-Free Path Planning with Constant TURNING RADIUS}

\section{A. Vehicle Model}

In this paper, front-wheel steering vehicles are considered and a rectangular model of the vehicle is assumed.

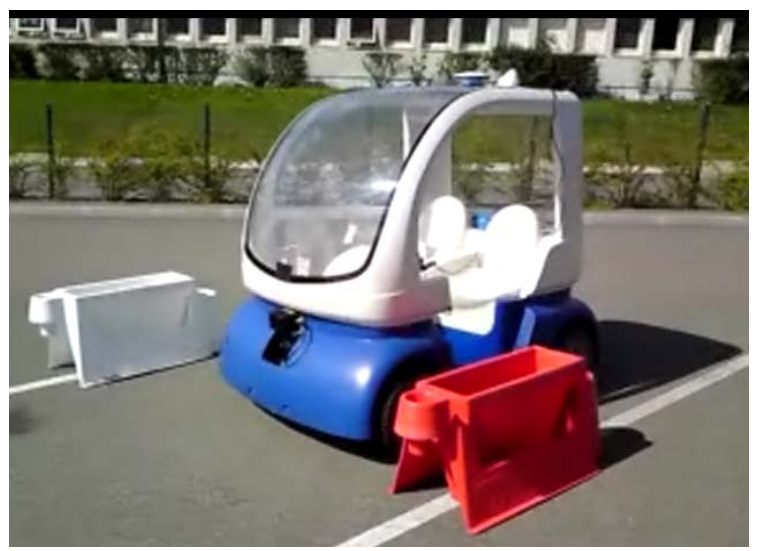

Fig. 1. The automatic CyCab vehicle developed at INRIA equiped with odometers, DGPS, and laser range finder 
To illustrate the results presented in this paper, we conduct simulation and experiments using a $\mathrm{CyCab}$ vehicle developed at INRIA, which is shown in Fig. 1. The vehicle parameters which affect the parking maneuver, as well as the parameter values used in the simulations, are presented in Table I.

TABle I. Vehicle Parameters

\begin{tabular}{|c|c|c|}
\hline Vehicle parameters & Notation & Value \\
\hline Longitudinal vehicle base & $l$ & $1.2 \mathrm{~m}$ \\
\hline Wheel base & $b$ & $1.2 \mathrm{~m}$ \\
\hline $\begin{array}{c}\text { Distance between the front axle and } \\
\text { the front bumper }\end{array}$ & $l_{1}$ & $0.35 \mathrm{~m}$ \\
\hline $\begin{array}{c}\text { Distance between the rear axle and } \\
\text { the rear bumper }\end{array}$ & $l_{2}$ & $0.35 \mathrm{~m}$ \\
\hline Maximum steering angle & $\alpha_{\max }$ & $\pi / 6 \mathrm{rad}$ \\
\hline
\end{tabular}

\section{B. Geometric Considerations for Collision-Free} Perpendicular Parking in One Maneuver

The geometry of the reverse perpendicular parking in one collision-free maneuver is shown in Fig. 2.

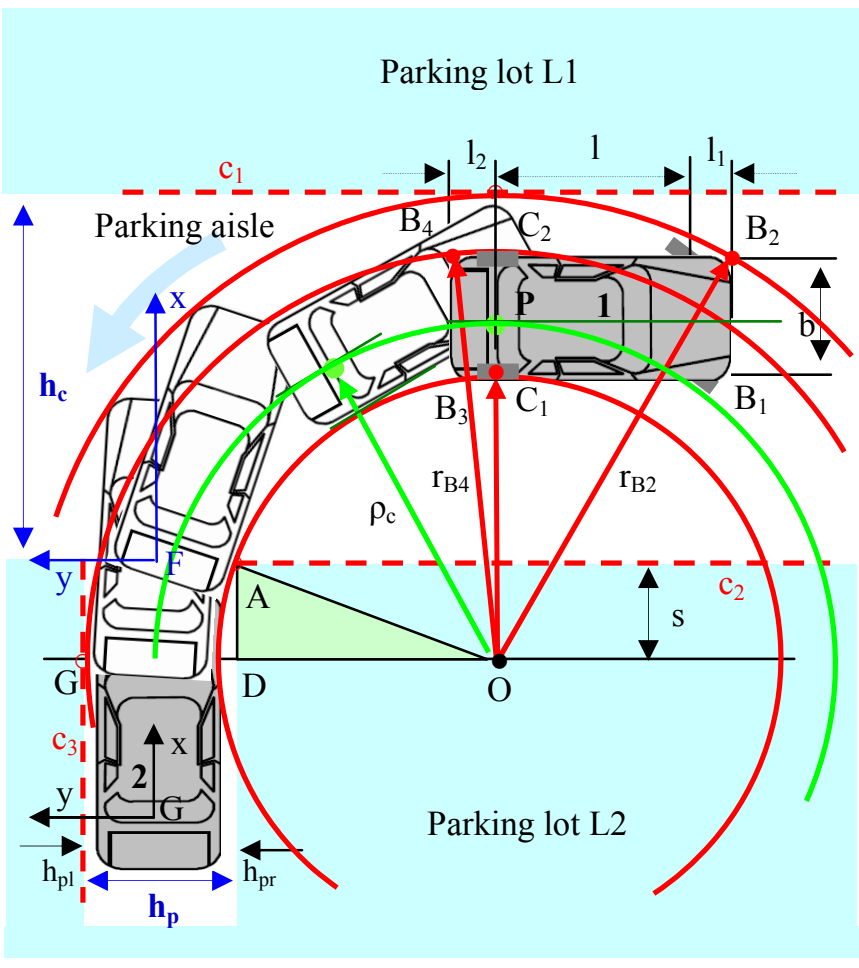

Fig. 2. Geometry of the collision-free perpendicular parking maneuver

Two inertial coordinate systems are assigned to the parking place: The frame $A x y$ with center $A$ which is placed in the middle between the borders of the parking space and which has its $F y$-axis aligned with the boundary $c_{2}$ of parking lot L2; the frame $G x y$ with center $G$ placed at the goal position of the parking place which has its $G x$-axis aligned with the $A x$-axis of Axy, as shown in Fig. 2.
1) Feasibility conditions for collision-free perpendicular parking in one maneuver

In the perpendicular parking scenario, the vehicle starts to move backward from an initial position 1 in the parking aisle, with constant steering angle $\alpha_{c}$ which is smaller or equal to the maximum steering angle $\alpha_{\max }$ and has to enter in the parking place without colliding with the boundary $c_{1}$ of parking lot $L 1$ and boundaries $c_{2}$, and $c_{3}$ of parking lot $L 2$. When a parallel orientation with respect to the parking place is attained, the vehicle continues to move backward in a straight line into the parking place until it reaches the final position 2 (Fig. 2).

Point $P$ placed in the middle of the rear wheel axle is assigned as the reference point of the vehicle (Fig. 2). Assuming a vehicle circular motion with center $O$ and turning radius $\rho_{c}\left(\rho_{c}=O P\right)$ calculated from the formula

$$
\rho_{c}=\frac{l}{\tan \alpha_{c}}
$$

The boundaries of the turning path during perpendicular parking are determined by the dimensions of the traces (circular arcs) formed by the left corner of the front bumper $B_{2}$ with radius $r_{B 2}$, the left corner of the rear bumper $B_{4}$ with radius $r_{B 4}$, and the end of the rear wheel axle $C_{l}$, as shown in Fig.2. Since the vehicle motion is rotation in the plane, the trajectories of these points form arcs of concentric circles.

From the $\triangle O C_{2} B_{2}$, applying the Pythagorean theorem, we obtain an expression for the radius $r_{B 2}$ of the circular arc traced by the left corner of the front bumper $B_{2}$ in terms of the vehicle parameters $l, l_{l}, b$ (Table I), and the constant turning radius $\rho_{c}$, as follows

$$
r_{B 2}=O B_{2}=\sqrt{\left(l+l_{1}\right)^{2}+\left(\rho_{c}+\frac{b}{2}\right)^{2}} .
$$

Similarly, from the $\triangle O C_{2} B_{4}$, we determine the radius $r_{B 4}$, of the circular arc traced by the left corner of the rear bumper $B_{4}$

$$
r_{B 4}=O B_{4}=\sqrt{l_{2}^{2}+\left(\rho_{c}+\frac{b}{2}\right)^{2}} .
$$

Let $O$ denotes the center of rotation (the Instantaneous Center of Rotation (ICR)) of the vehicle when it starts the parking maneuver with constant front-wheel steering angle $\alpha_{c}$. Depending on the sign of the $x$-coordinate of ICR $O$ with respect to coordinate frame $A x y$, i.e., the offset $s$ (Fig. 2), different formulas can be derived in order to determine the required width $h_{p}$ of the parking place and the width of the parking aisle (the corridor) $h_{c}$, in order to ensure collision-free perpendicular parking in one maneuver. Due to a limited space, in this paper, we consider the case when $s$ is negative and belongs to the interval $s \in\left[-\left(\rho_{c}-\frac{b}{2}\right), 0\right]$, which is the case when the dimension of the parking aisles and the parking place 
are not too large. The lower value corresponds to the case when the right side of the vehicle $B_{1} B_{3}$ (Fig.2) lies on the boundary line $c_{2}$ of parking lot $L 2$.

In order to avoid collision between the left corner $B_{2}$ of the front bumper with the boundary $c_{1}$ of $L 1$ (Fig. 2), using (2), we obtain an expression for the width of the parking aisle $h_{c}$, as follows

$$
h_{c}=r_{B 2}-|s|=\sqrt{\left(l+l_{1}\right)^{2}+\left(\rho_{c}+\frac{b}{2}\right)^{2}}-|s| \text {. }
$$

The function $h_{c}=\mathrm{f}(s)$ is linear in $s$, positive and monotonically increasing in the above-mentioned closed interval for $s$. Therefore, it takes its minimum and maximum values at the ends of this interval.

To avoid collision between the right point $C_{I}$ of the rear axle with vertex $A$ of the obstacle $L 2$, from the $\triangle O A D$, applying the Pythagorean Theorem, the distance $O D$ is determined as follows

$$
O D=\sqrt{\left(\rho_{c}-\frac{b}{2}\right)^{2}-s^{2}}
$$

In order to avoid collision between the left corner $B_{4}$ of the rear bumper with the edge $c_{3}$ of the parking place, using (3) and (5), the following expression for the width of the parking space $h_{p}$, is obtained

$$
h_{p}=r_{B 4}-O D=\sqrt{l_{2}^{2}+\left(\rho_{c}+\frac{b}{2}\right)^{2}}-\sqrt{\left(\rho_{c}-\frac{b}{2}\right)^{2}-s^{2}} .
$$

Consider the function $h_{p}=\mathrm{f}(s)$, which is continuous on the closed interval of $s$ mentioned above. The function is differentiable on the open interval $s \in\left(-\left(\rho_{c}-\frac{b}{2}\right), 0\right)$, and its derivative is

$$
\frac{\partial h_{p}}{\partial s}=\frac{s}{\sqrt{\left(\rho_{c}-\frac{b}{2}\right)^{2}-s^{2}}}<0 .
$$

Therefore $h_{p}=\mathrm{f}(s)$ is strictly decreasing on the closed interval $\left[-\left(\rho_{c}-b / 2\right), 0\right]$. The maximum and minimum values of $h_{p}$ can be found by replacing the limit values $s=-\left(\rho_{c}-b / 2\right)$ and $s=0$ of the interval, respectively, in (6).

2) Collision-free parking with assigned values for the widths of the parking aisle and the parking place

From a practical point of view, it is important to determine the starting positions of the vehicle for automatic parking without collision in one maneuver, in the case, when the widths $h_{c}$ and $h_{p}$ of the parking aisle and the parking space, are specified in advanced. Suppose that the widths of the parking aisle and the parking place are set as $h_{c}=h_{c d}$ and $h_{p}=h_{p d}$, respectively, and also that $h_{c d}<r_{B 2}$. In this case, from (2) and (4), it follows that

$$
-|s|_{\max }=h_{c d}-r_{B 2} .
$$

From (3) and (6), we obtain a formula for the minimum value of $s$ as follows

$$
-|S|_{\min }=-\sqrt{\left(\rho_{c}-\frac{b}{2}\right)^{2}-\left(r_{B 4}-h_{p d}\right)^{2}} .
$$

It should be noted that, when $s=-|\mathrm{s}|$ min the vehicle will park without collision, but asymmetrically (not centered in the middle) in the parking space with respect to its boundaries, i.e., $h_{p l} \neq h_{p r}$ (Fig.2). When the vehicle is parallel to the parking space, the distances between the car and the parking space boundaries $h_{p l}$ and $h_{p r}$ are determined as follows

$$
\begin{gathered}
h_{p r}=\left(\rho_{c}-\frac{b}{2}\right)-\sqrt{\left(\rho_{c}-\frac{b}{2}\right)^{2}-s^{2}}, \\
h_{p l}=h_{p d}-b-h_{p r} .
\end{gathered}
$$

3) Symmetrical collision-free parking with assigned values for the widths of the parking aisle and the parking place

From a practical view point, it is better to park the car symmetrically with respect to the boundaries of the parking place, since it is not very wide. For this end, we calculate the minimum value of the offset $s=s_{m}$, in order to park symmetrically the vehicle in the center of the parking space (Fig. 2). We set

$$
h_{p s}:=h_{p r}=h_{p l}=\frac{h_{p d}-b}{2} .
$$

From the $\triangle O A D$ (Fig. 3), the distance $O D$ is determined as

$$
O D=\sqrt{\left(\rho_{c}-\frac{b}{2}\right)^{2}-s_{m}^{2}}
$$

Since the turning radius can be expressed as

$$
\rho_{c}=\frac{b}{2}+h_{p r}+O D
$$

and substituting $h_{p r}$ from (10) and $O D$ from (13) into (14), we arrive to an expression for $s_{m}$, as follows 


$$
-|s|_{m}=-\sqrt{\left(\rho_{c}-\frac{b}{2}\right)^{2}-\left(\rho_{c}-\frac{h_{p d}}{2}\right)^{2}} .
$$

The new offset $-|\mathbf{s}|_{\mathrm{m}}$ is bigger than minimum offset $-|\mathbf{s}|_{\text {min }}$ given by (9), (-|S|$\left.\left.\right|_{m}>-|s|_{\text {min }}\right)$. In general, it must be checked whether the new offset $-|\mathrm{s}|_{\mathrm{m}}$ is smaller than $-|\mathrm{s}|_{\max }$ given by (8). If it is the case, the car can park symmetrically without collision in reverse with a minimum offset $s=-|\mathrm{s}|_{\mathrm{m}}$. In this case, however, the boundary $c_{3}$ of the parking place will not be tangent to the arc of circle traced by point $B_{4}$ of the left corner of the rear bumper; nevertheless, point $A$ (vertex $A$ of obstacle L2) will lie again on the arc of circle traced by point $C_{l}$ of the rear vehicle axle. Therefore, given specified values $h_{c}=h_{c d}$ and $h_{p}=h_{p d}$, the offset $s$ can take values in the closed interval $|\mathbf{s}| \in\left[-|\mathbf{s}|_{\mathrm{m}},-|\mathbf{s}|_{\max }\right], \quad$ where $-|\mathbf{s}|_{\mathrm{m}}$ and $-|\mathbf{s}|_{\max }$ are determined by formulas (15) and (8), respectively.

Hence, in order to perform reverse perpendicular parking without collision in one maneuver and to place the vehicle symmetrically into the parking place with assigned constant steering angle $\alpha_{c}$ which is smaller or equal to the maximum steering angle $\alpha_{\max }$, the starting position, i.e., the reference point $P$ of the vehicle has to be on any one of the arcs of circles with radius $\rho$ of center $O\left(x_{O}, y_{O}\right)$ with respect to the coordinate system Fxy attached to the parking place (Fig.2), where $x_{O}$ belongs to the interval $\left[-|\mathrm{s}|_{\mathrm{m}},-|\mathrm{s}|_{\max }\right]$ and $y_{O}=-\rho_{c}$. The vehicle initial orientation has to be tangent to this arc. In particular, if the orientation of the vehicle is (almost) perpendicular to the parking place, the initial coordinates of the reference point $P$ with respect to the frame $F x y$ have to be $x_{P}(0)=\rho-|s|$, where $|s| \in\left[-|\mathrm{s}| \mathrm{m},-|\mathrm{s}|_{\max }\right]$ and $y_{P}(0)=-\rho_{c}$. The reference path of the parking maneuver consists of two parts. The first one is a circular arc with center $O$ connecting the staring position of the vehicle and the $F x$-axis of Fxy, which is tangent to the circular arc. At the tangent point $T$ with coordinates $(-|s|, 0)$, the car will be parallel to the parking place. The second part of the reference path is a straight line with distance $T G$ along the $F y$ axis of the coordinate frame $F x y$ between the tangent point $T$ and the goal position $G$ of the parking place, since point $G$ lies on the $F x$-axis of $F x y$.

\section{STEERING CONTROL}

For a low speed motion, which is the case of the parking maneuver, we assume that the wheels of the vehicle roll without sliding, and the velocity vectors are in the direction of the orientation of the wheels. We consider a simplified (bicycle model) of the vehicle, where the front and rear wheels are replaced by two virtual wheels, placed at the longitudinal axis of the vehicle. The inertial coordinate system $G x y$ is attached to the parking place in the goal position $G$ (Fig. 2). The coordinates of the reference point $P$ in $G x y$ are denoted by $\left(x_{P}\right.$, $\left.y_{P}\right)$. The orientation of the vehicle $\theta$ is defined as an angle between the $x$-axis of $G x y$ and the longitudinal vehicle base. Information from the encoders mounted on the wheels was used for estimation the position of the vehicle in the parking area. The steering angle of the front virtual wheel is denoted by $\alpha$. The equations of motion of the vehicle in the plane have the form [7]

$$
\begin{aligned}
& \dot{x}_{P}=v_{P} \cos \theta \\
& \dot{y}_{P}=v_{P} \sin \theta, \\
& \dot{\theta}=\frac{v_{P}}{l} \tan \alpha
\end{aligned}
$$

where $v_{P}$ is the velocity of point $P$. We consider a practical stabilization of the vehicle in the parking place. Our approach is based on controlling the motion of the vehicle along a straight line (the $G x$-axis of $G x y$ ) passing through the goal point $G$ in the parking place and aligned with the orientation of the place. The velocity profile is dependent of the distance between the vehicle and the goal position [7]. Since the reference path for the first part of the parking maneuver is a circular arc, a bang-bang feedback controller [8], where the front wheel steering angle is constrained by magnitude and takes only two constant values may be a solution, since the vehicle trajectories will represent circular arcs. However, in practice, due to the discontinuity of the bang-bang control law, an undesirable behavior of the system (chattering) will occur. In order to avoid the chattering, in this paper, a saturated control based on hyperbolic tangent function is proposed, which is also constrained by magnitude, but the control function is continuous.

\section{A. Saturated Control}

In this paper, we propose a differentiable saturation in the form of hyperbolic tangent $(\tanh ()$.$) constraint. This function$ is bounded by \pm 1 . Also $\tanh (x) \geq 0$ if $x \geq 0$, and $\tanh (x)<0$ if $\mathrm{x}<0 . \operatorname{Tanh}(x)$ is close to the signum function, when in $\tanh \left(K_{t} x\right)$ the gain $K_{t}$ is large, as shown in Fig. 3.

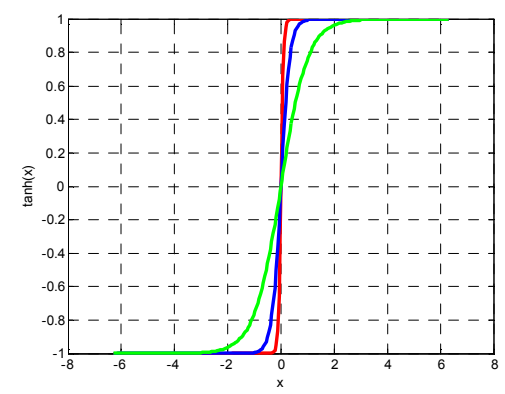

Fig. 3. The function $\tanh \left(K_{t} x\right)$ for $K_{t}=1,3$, and 10 .

The proposed bounded controller has the form

$$
\alpha=a \tan \left[l u \tanh \left(K_{t} v\right)\right]
$$

where

$$
v=K\left(\theta-a_{0} y_{P}\right),
$$

$$
u=\frac{\tan \alpha_{c}}{l}
$$


and $K_{t}, K$ and $a_{0}$ are positive constants. The proposed controller achieves asymptotic stability of the closed loop system composed of equations (16)-(19). The proof is similar to those presented in [7] and is omitted here due to space limitation.

It should be noted that the constant value $\alpha_{c}$ for the front wheel steering angle can be smaller or equal of its maximum value $\alpha_{\max }$. If the starting position of the vehicle does not satisfy the conditions mentioned in Section II for parking in one maneuver, multiple maneuvers have to be performed in order to reach the goal position in the parking place.

\section{SimUlation RESUlts}

Simulation results using MATALAB are presented to illustrate the effectiveness of the geometrical path planning procedure for collision-free perpendicular parking in one maneuver, as well as the performance of the proposed saturated steering controller for practical stabilization of the vehicle into the parking place. The parameters of the test vehicle (CyCab) are given in Table I.

\section{A. Path Planning Simulation}

In order to demonstrate the proposed path planning procedure for collision-free perpendicular parking in one maneuver presented in Section II, in the first simulation, we present the relationships between the widths $h_{c}$ and $h_{p}$ of the parking aisle and the parking space, as functions of the offset $s$ which belongs to the interval $\left[-\left(\rho_{c}-b / 2\right), 0\right]$ where $s \leq 0$ using parameters of the test vehicle with $\alpha_{c}=\alpha_{\max },\left(\rho_{c}=\rho_{\min }\right)$. The assigned values of $h_{c}$ and $h_{p}$ were chosen to be: $h_{c d}=3 m$ and $h_{p d}=2 m$.

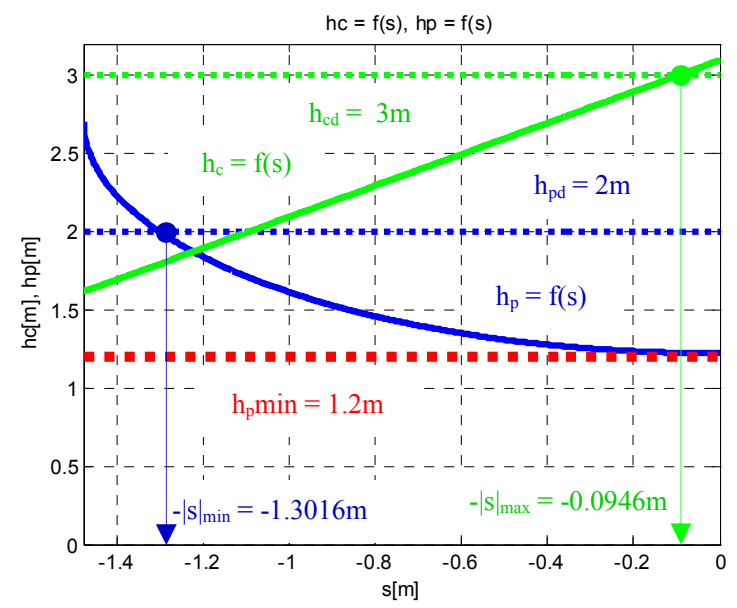

Fig. 4. Collision-free interval for $s$

As seen from Fig.4, the function $h_{p}=\mathrm{f}(s)$ (the solid blue line) decreases in the interval and converges to $b=1.2 m$ (the red dotted line), which is exactly the length of the wheel base of the vehicle. Meanwhile, the graph intersects the horizontal line for the assigned value of $h_{p d}=2 m$ (the blue dotted line) at $s=-$ $|\mathrm{s}|_{\min }=-1.3016 \mathrm{~m}$, which is the minimum value of $s$ obtained from (9) for collision-free parking. In order to park the vehicle in one maneuver for $s=-|\mathrm{s}|_{\min }=-1.3016 m$, from (4), the required minimum width $h_{c}$ of the parking aisle has to be $h_{c}=$ $1.793 \mathrm{~m}$ which is less than the specified value of $h_{c d}=3 \mathrm{~m}$.

The function $h_{c}=\mathrm{f}(s)$ (the green solid line) increases linearly in this interval and the graph intersects the horizontal line for the assigned value of $h_{c d}=3 m$ (the green dotted line) at $s=-|\mathrm{s}|_{\max }=-0.0946 \mathrm{~m}$, which is the maximum value of $s$, obtained from (8). For $s=-|\mathrm{s}|_{\max }=-0.0946 m$, from (6), the required minimum width $h_{p}$ of the parking place has to be $h_{p}=$ $1.2258 \mathrm{~m}$, which is less than the specified value of $h_{p d}=2 \mathrm{~m}$.

Therefore, given specified values $h_{c}=h_{c d}=3 m$ and $h_{p}=h_{p d}$ $=2 \mathrm{~m}$ for the parking aisle and the parking space, respectively, for collision-free parking, the offset $s$ can take values in the interval $\left[-|\mathrm{s}|_{\min },-|\mathrm{s}|_{\max }\right]=[-1.3016,-0.0946]$, where the boundary values are determined by (9) and (8), respectively.

For $s=-|\mathrm{s}|_{\min }$, the vehicle will park without collision but not centered in the middle of the parking place. In this case, Using (10) and (11), simulation results indicate that the distances between the car and boundaries of the parking place are $h_{p r}=0.7772 \mathrm{~m}$ and $h_{p l}=0.0228 \mathrm{~m}$ for $h_{p d}=2 \mathrm{~m}$. In order to park symmetrically in the parking place $\left(h_{p r}=h_{p l}=0.4 m\right)$, by using (15), the minimum offset $-\left|s_{m}\right|$ was obtained to be $-\left|s_{m}\right|=-$ $1.0113 m$, and this value is bigger than $-|\mathrm{s}|_{\min }=-1.3016,\left(-|\mathrm{s}|_{\mathrm{m}}>\right.$ $-|\mathrm{s}|_{\min }$ ), obtained from (9), but smaller than - $|\mathrm{s}|_{\max }=-0.0946 \mathrm{~m}$. Therefore, given specified values $h_{c d}=3 m$ and $h_{p d}=2 m$, for centered parking into the parking place, the offset $s$ can take values in the closed interval $-|\mathrm{s}| \in\left[-|\mathrm{s}|_{\mathrm{m}},-|\mathrm{s}|_{\max }\right]=[-1.0113,-$ 0.0946].

\section{B. Simulation of Perpendicular Parking using Saturated Control}

Simulation results are presented to illustrate the performance of the proposed saturated steering controller for perpendicular reverse parking. Using (1), for $\alpha_{c}=\alpha_{\max }$, for the minimum turning radius $\rho_{c}=\rho_{\text {min }}$ is obtained $\rho_{\text {min }}=2.0785 \mathrm{~m}$. The dimensions of the parking aisle and the parking place were the same used before: $h_{c d}=3 m$ and $h_{p d}=2 m$.

1) Simulations for perpendicular parking in one maneuver

The initial coordinates of the vehicle reference point $P$ with respect to the inertial frame $G x y$ attached to the goal position were chosen to be $\left(x_{P}(0), y_{P}(0)\right)=(3 m,-\rho)=(3 m,-2.07 m)$ where $y_{P}(0)=-\rho_{\text {min }}$. In this case the offset $-|s|=-1.0785 m \in[-$ $\left.|\mathrm{s}|_{\mathrm{m}},-|\mathrm{s}|_{\max }\right]=[-1.3016,-0.0946]$. The initial orientation of the vehicle is chosen to be $\theta(0)=-\pi / 2 \mathrm{rad}$. Therefore, the vehicle is able to park in one maneuver. The maximum value of the timevariant vehicle velocity was $\left|v_{P}\right|=0.3 \mathrm{~m} / \mathrm{s}$. The values of the saturated tanh-type controller were $K_{t}=8, K=5.85, a_{0}=0.17$.

The planar path of the vehicle (a) and an animation of the perpendicular reverse parking in one maneuver are presented in Fig. 5.Evolution in time of the vehicle velocity (a) and the front-wheel steering angle (b) are presented in Fig. 6. Since the saturation control is continuous, there is not chattering when the position of the vehicle is in the vicinity of the tracking line and in addition, the orientation error is small too. As seen from the simulation results (Fig. 6,b), the saturated control can be used instead of bang-bang control in order to steer the vehicle into the parking place without collision, since it is close to the signum function when the gain $K_{t}$ is large enough. 

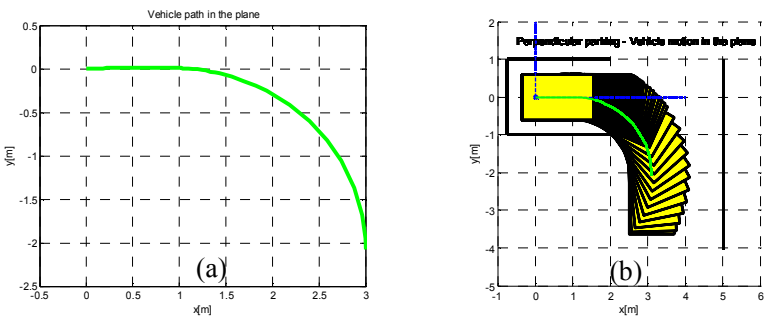

Fig. 5. Perpendicular parking in one maneuver: Planar paths of the vehicle (a) and animation of the parking maneuver (b)
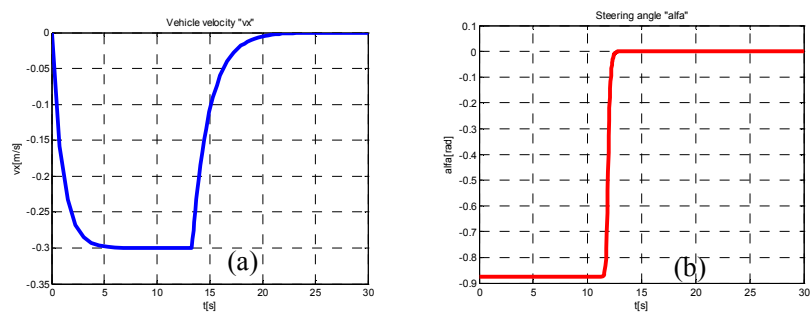

Fig. 6. Perpendicular parking in one maneuver: Evolution in time of the vehicle velocity (a) and the front-wheel steering angle (b)

\section{2) Simulations for perpendicular parking in multiple} maneuvers

In this case, the initial coordinates of the vehicle reference point $P$ with respect to the inertial frame $G x y$ attached to the goal position were chosen to be $\left(x_{P}(0), y_{P}(0)\right)=(3 m,-(\rho+0.5))$ $=(3 m,-1.57 m)$. The offset $-|s|=-1.0785 m$ belongs to the interval $\left[-|\mathrm{s}|_{\min },-|\mathrm{s}|_{\max }\right]=[-1.3016,-0.0946]$, but $y_{P}(0) \neq-\rho_{\min }$ and the vehicle cannot park in one maneuver. The initial orientation of the vehicle was chosen to be $\theta(0)=-\pi / 2 \mathrm{rad}$. In this case, the vehicle cannot park in one maneuver and multiple maneuvers are necessary. The values of the controller gains were like in the first simulation for automatic parking in one maneuver. The planar path of the vehicle (a) and an animation of the perpendicular reverse parking in three maneuvers are presented in Fig. 7. The overall parking maneuver was achieved by three consecutive maneuvers (backward-forwardbackward).
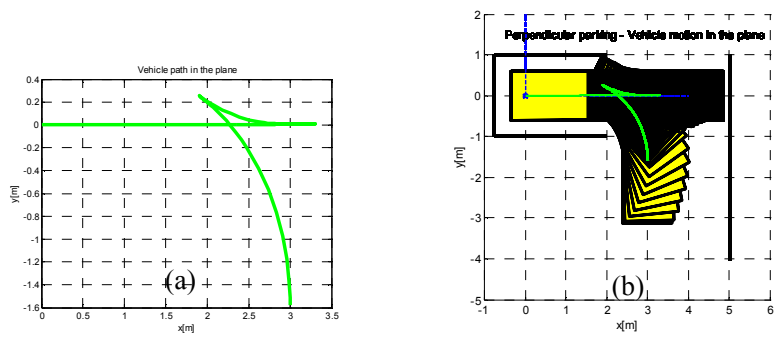

Fig. 7. Perpendicular parking in three maneuvers: Planar paths of the vehicle (a) and animation of the parking maneuver (b)

Evolution in time of the vehicle velocity (a) and the frontwheel steering angle (b) are presented in Fig. 8.

The saturated controller has been implemented on an experimental automatic electric vehicle $\mathrm{CyCab}$ and initial tests of perpendicular reverse parking in one maneuver have been initialized (Fig. 1). In the first tests, only information from the encoders mounted on the wheels were used for estimation of the the position and localisation of the vehicle in the parking area. First experiments confirm the effectiveness of the proposed controller.
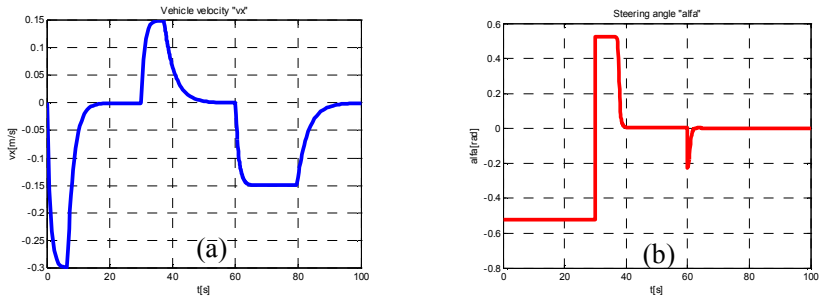

Fig. 8. Perpendicular parking in three maneuvers: Evolution in time of the vehicle velocity (a) and the front-wheel steering angle (b)

\section{CONCLUSION}

In this paper, the problem of perpendicular collision-free reverse parking of front wheel steering vehicles has been considered. Geometric considerations for collision-free perpendicular parking in one reverse maneuver have been first presented, where the shape of the vehicle and the parking environment have been expressed as polygons. Relationships between the widths of the parking aisle and parking place, as well as the parameters and the initial position of the vehicle have been given, in order to plan a collision-free maneuver, in the case, when the car has to be centered into the parking place. A tanh-type steering controller for straight-line tracking has been proposed and evaluated. It was demonstrated that this controller which is continuous, was able to achieve quick steering avoiding chattering and can be successfully used in solving parking problems. Simulation results, as well as the first experimental tests with an automatic $\mathrm{CyCab}$ vehicle confirm the effectiveness of the proposed control scheme.

\section{REFERENCES}

[1] USAF - LANDSCAPEDESIGN GUIDE, available at: http://www.ttap. mtu.edu/publications/2007/ParkingDesignConsiderations.pdf.

[2] B. Gutjahr and M. Werling, Automatic collision avoiding during parking maneuver - an optimal control approach, In Proc. 2014 IEEE Intel.. V. Symposium, 2014, pp. 636-641.

[3] S. Blackburn, The geometry of perfect parking, Available at: http://personal.rhul.ac.uk/uhah/058/perfect_parking.pdf.

[4] C. Pradalier, S. Vaussier and P. Corke, Path planning for parking assistance system : Implementation and experimantation, In Proc. Austr. Conf. Rob. Automation, 2005.

[5] J. Moon, I. Bae, J. Cha, and S. Kim, A trajectory planning method based on forward path generation and backward tracking algorithm for automatic parking systems, In Proc. IEEE Int. Conf. Intell. Transp. Systems, 2014, pp. 719-724.

[6] K. Lee, D. Kim, W. Chung, H. Chang, and P. Yoon, Car parking control using a trajectory tracking controller, In Proc SICE ICASE Int. J. Conference, 2006, pp. 2058-2063.

[7] P. Petrov and F. Nashashibi, Saturated feedback control for an automated parallel parking assist system, In Proc. IEEE Conf. Contr. Autom. Rob. Vision, 2014, pp. 577-582.

[8] P. Petrov, C. Boussard, S. Ammoun, and F. Nashashibi, A hybrid control for automatic docking of electric vehicles for recharging, In Proc. IEEE Int. Conf. Rob. Automation, 2012, pp. 2966-2971.

[9] Avalable at: https:// www.youtube.com/watch?v=YbrwUrxFybQ

[10] Avalable at: https://www.youtube.com/watch?v=b_m8DqTlOLE 